\title{
Upregulation of BAG3 with apoptotic and autophagic activities in maggot extract-promoted rat skin wound healing
}

\author{
JIAN-LI DONG ${ }^{1}$, HAI-CAO DONG ${ }^{1}$, LIANG YANG ${ }^{1}$, ZHE-WEN QIU $^{2}$, JIA LIU $^{3}$, HONG LI $^{3}$, \\ LI-XIA ZHONG ${ }^{4}$, XUE SONG $^{2}$, PENG ZHANG $^{2}$, PEI-NAN LI ${ }^{1}$ and LIAN-JIE ZHENG ${ }^{1}$
}

\author{
${ }^{1}$ Department of Orthopedic Surgery, Second Clinical College, Dalian Medical University, Dalian, Liaoning 116011; \\ ${ }^{2}$ Experimental Animal Center, Dalian Medical University; ${ }^{3}$ Department of Cell Biology, College of Basic Medical \\ Sciences, Dalian Medical University, Dalian, Liaoning 116044; ${ }^{4}$ Department of Oncology, Guangzhou University \\ of Chinese Medicine, Guangzhou, Guangdong 510000, P.R. China
}

Received March 24, 2017; Accepted September 21, 2017

DOI: $10.3892 / \mathrm{mmr} .2017 .8331$

\begin{abstract}
Maggot extract (ME) accelerates rat skin wound healing, however its effect on cell maintenance in wound tissues remains unclear. B-cell lymphoma (Bcl) 2-associated athanogene (BAG)3 inhibits apoptosis and promotes autophagy by associating with Bcl-2 or Beclin 1 . Bcl-2, the downstream effector of signal transducer and activator of transcription 3 signaling, is enhanced in ME-treated wound tissues, which may reinforce the Bcl-2 anti-apoptotic activity and/or cooperate with Beclin 1 to regulate autophagy during wound healing. The present study investigated expression levels of BAG3, Bcl-2, Beclin 1 and light chain (LC)3 levels in rat skin wound tissues in the presence and absence of ME treatment. The results revealed frequent TUNEL-negative cell death in the wound tissues in the early three days following injury, irrespective to ME treatment. TUNEL-positive cells appeared in the wound tissues following 4 days of injury and $150 \mu \mathrm{g} / \mathrm{ml} \mathrm{ME}$ efficiently reduced apoptotic rate and enhanced BAG3 and Bcl-2 expression. Elevated Beclin 1 and LC3 levels and an increased LC3 II ratio were revealed in the ME-treated tissues during the wound healing. The results of the present study demonstrate the anti-apoptotic effects of BAG3 and Bcl-2 in ME-promoted wound healing. Beclin 1/LC3 mediated autophagy may be favorable in maintaining cell survival in the damaged tissues and ME-upregulated BAG3 may enhance its activity.
\end{abstract}

Correspondence to: Dr Pei-Nan Li or Professor Lian-Jie Zheng, Department of Orthopedic Surgery, Second Clinical College, Dalian Medical University, 467 Zhong-Shan Street, Dalian, Liaoning 116011, P.R. China

E-mail: zhenglianj@sina.cn

E-mail: delaho@126.com

Key words: maggot extract, rat skin wound model, BAG3, Bcl-2, Beclin 1, apoptosis, autophagy

\section{Introduction}

Skin wounds are the commonest body damage in clinics, and their consequences are largely determined by the quality of wound healing (1). Wound healing is the process by which the damaged tissue is repaired after trauma through an orchestrated cascade of stepwise biochemical events including blood clotting with inflammation, tissue growth (proliferation) and tissue remodeling (maturation) $(2,3)$. Cell turnover happens in those steps, including the removal of the dysfunctional cells from the wound bed in the forms of necrosis (4) and/or apoptosis $(5,6)$, the activation of cell proliferation machinery to repaire the lost tissue $(7,8)$ and the remodeling of the regenerated tissue by inducing apoptosis of unneeded cells (9). Apparently, a reagent that well regulates cell death and proliferation would be of practical values in better management of skin wounds.

Multiple molecular factors involve in skin wound healing, of which the signal transduction pathways mediated by Smad/TGF $\beta(10,11)$ and STAT3 $(11,12)$ play active roles through initiating or upregulating the expression of a series of growth-promoting genes including anti-apoptotic genes such as Survivin and Bcl-2 (13). It has been recognized that the biological effects of $\mathrm{Bcl}-2$ can be enhanced via interaction with BAG3 (BCL2-associated athanogene 3) (14-16). For instance, combined inhibition of Bcl-2 and BAG3 overcomes apoptotic resistance in glioblastoma in vitro and in vivo (15) and significant decrease of BAG3 expression leads cardiac cells to apoptosis (17). On the other hand, BAG3 promotes autophagy through interacting with autophagy-associated protein Beclin 1 (18) because an increased level of BAG3 results in stimulation of autophagy in glioblastoma cells (19). Although extensive cell turnover including apoptosis and autophagy occurs in the skin wound tissues, the involvement and potential role(s) of BAG3 in stepwise wound healing remains unknown.

It has long been recognized that maggot can be used to accelerate skin wound healing in the manners of eating unhealthy tissues and secreting/eliminating the bioactive products $(20,21)$. Our recent results demonstrate that the extract isolated from maggots without secretion and elimination efficiently shortens the wound closure time by enhancing Smad2/TGF $\beta$ and 
STAT3 signaling activities, respectively (22). Because Bcl-2 is one of the downstream effector of STAT3 signaling (23) and its function is enhanced by associating with BAG3 co-chaperone protein $(24,25)$, BAG3 expression pattern in wound tissue may be altered by maggot extract and may functionally influence the apoptotic pathway and autophagy-promoting activity of Beclin 1 in the post-trauma regenerating tissues. To address these issues, BAG3, BCL-2, Beclin 1 and LC3 levels in rat skin wound tissues without and with maggot extract treatment are analyzed and their relevance with the rates of wound healing and cell proliferation are evaluated.

\section{Materials and methods}

Maggot extract preparation. The maintenance of Lcuprina blowflies and their larvae, the ways of maggot collection and treatment and the method for maggot extract preparation were conducted in the manners described elsewhere (22). We found that the full maggot extract (tissue lysate + excretion/secretion) exerted the best repair promoting effects on wound tissues in comparison with that of the mixture of excretion/secretion (ES) and the tissue lysate prepared from the maggots after excretion and secretion and the optimal working concentration was $150 \mu \mathrm{g} / \mathrm{ml}$ (22). Therefore, the vaseline-diluted full maggot extracts in the concentration of $150 \mu \mathrm{g} / \mathrm{ml}$ was adopted to dress the wound beds.

Statement of assurance of proper animal experiments. The protocol of animal study was designed in compliance with the National Research Council's criteria for humane care as outlined in 'Guide for the Care and Use of Laboratory Animals' prepared by the Institute of Laboratory Animal Resources and published by the National Institutes of Health (NIH Publication No. 86-23, Revised 1985). Before conducting the experiments, the contents of the present study were reviewed and approved by the ethical and animal warfare committee of Dalian Medical University. During the experiments, all animals received humane care. When the experiments were finished, they were returned to the institutional animal center without sacrificing.

Rat wound model. After getting the permission to conduct the animal experiment from Institutional Ethics Committee and the Committee on Research Animal Care of Dalian Medical University, sixteen 10-week old male Sprague Dawley rats were provided by the Experimental Animal Center of Dalian Medical University and reared under specific pathogen-free/SPF condition. The rats were anaesthetized with $12 \mathrm{mg} / \mathrm{kg}$ xylazine via intraperitoneal injection. A pair of $2 \mathrm{~cm}$ diameter round open wounds down to the muscle fascia was made on the left for the purposes of sequential biopsy and on the right flanks for wound area measurement, respectively (26). The animals were randomly divided into two experimental groups of 8 animals/group as Group 1 (G1): Dressed with vaseline only as untreated control; G2: Treated with a mixture of vaseline and $150 \mu \mathrm{g} / \mathrm{ml}$ full maggot extract (without excretion and secretion). The treatments lasted for 16 days by daily dressing the reagents. The margins of individual wounds were outlined in regular changed red and black colors at day $1,3,4,6,8,10,12,14$ and 16 by directly placing a transparency model sheet on the wounds on the right flanks (12). The areas enclosed by the traced wound margins of the two experimental groups were calculated by Digital-transparency wound area measurement (12). The animal experiments were restrictively followed the guidelines of the Association for Assessment and Accreditation of Laboratory Animal Care, International and repeated for three times for establishing statistical significance.

Wound tissue biopsy. The tissues in the size of $0.3 \times 0.3 \times 0.2 \mathrm{~cm}$ were biopsied from the margins and beds of the wounds on the left flanks at the post-trauma times of day 1 , day 4 , day 7 , day 12 and, if available, day 16 or day 3, day 5, day 9 and, if available, day 14 . The biopsy was sequentially conducted at the 3, 6, 9 and 12 o'clock positions of the round wounds. The collected tissues were snap frozen in liquid nitrogen and stored at $-80^{\circ} \mathrm{C}$ until use. The frozen tissues were vertically sectioned in $5 \mathrm{~mm}$ thickness into $80-100$ pieces which were immediately put into $40 \mu \mathrm{l}$ cell lysate buffer for protein isolation (27). The remaining parts of the sample tissues were sectioned in $7 \mu \mathrm{m}$ thickness for histological and immunohistochemical staining.

TUNEL apoptosis assay. Terminal deoxynucleotide transferase (TdT)-mediated dUTP-biotin nick-end labeling (TUNEL) assay was employed to detect apoptotic cells according to producer's instructions (Promega Corp., Madison, WI, USA). Briefly, the tissue samples were pre-incubated with $3 \%$ bovine serum albumin (BSA) in PBS for $30 \mathrm{~min}$ at RT to prevent nonspecific labeling and then incubated for $1 \mathrm{~h}$ at $37^{\circ} \mathrm{C}$ in a humid chamber with the TUNEL mixture containing 0.13 5 Uipl calf thymus TdT. After three 5 min washes with PBS at room temperature, $100 \mu \mathrm{l}$ anti-FITC-AP conj. was applied on each sample for $30 \mathrm{~min}$ at $37^{\circ} \mathrm{C}$. After resaturation in blocking reagent, the tissues were treated for $1 \mathrm{hr}$ at room temperature with a 1:120 diluted peroxidase-labeled anti-digoxigenin sheep Fab fragment, followed by $0.05 \%$ 3,3'-diaminobenzidine tetrahydrochloride (DAB) color reaction.

Immunohistochemical staining. The statuses of BAG3, Bcl-2, Beclin 1 and LC3 expression in the wound tissues were analyzed immunohistochemical staining by the method described elsewhere (28). The antibodies against those target proteins were purchased from Santa Cruz Biotechnology, Inc., Santa Cruz, CA, USA. Color reaction was developed using 3, 3'-diaminobenzidine tetrahydrochloride (DAB). The samples without first antibody incubation were cited as negative control.

Western blot analysis. To validate the immunohistochemical results, total cellular proteins were prepared from the wound tissues without and with maggot extract treatment by the method described elsewhere (12). The sample proteins $(50 \mu \mathrm{g} /$ lane $)$ were separated in $10 \%$ sodium dodecylsulfate-polyacrylamide gel electrophoresis and transferred to polyvinylidene difluoride membrane (Amersham, Buckinghamshire, UK). The membrane was blocked with $5 \%$ skimmed milk in TBS-T $(10 \mathrm{mM}$ Tris-HCl, $\mathrm{pH} 8.0,150 \mathrm{mM} \mathrm{NaCl}$ and $0.5 \%$ Tween 20) at $4^{\circ} \mathrm{C}$, rinsed $10 \mathrm{~min}$ for three times with TBS-T, followed by $3 \mathrm{~h}$ incubation at room temperature with the first antibody and then $1 \mathrm{~h}$ incubation with HRP-conjugated anti-mouse 
or anti-rabbit IgG (Zymed Lab, Inc., San Francisco, CA, USA). The bound antibody was detected using the enhanced chemiluminescence system (Roche GmbH, Mannheim, Germany). After removing the labeling signal by incubation with stripping buffer, the membrane was reprobed with other antibodies one by one until all of the parameters were examined. The parameters checked are in parallel with that of immunohistochemical staining. The results of western blotting were quantified by densitometry analysis, using Quantity One software according to producer's instruction (Bio-Rad Lab., Berkeley, CA, USA). $\beta$-actin bands of individual samples were cited as internal quantitative control.

Statistical analysis. The wound healing statuses of individual experimental groups at different tracing times and MTT data were evaluated by the independent-samples t-test and one-way ANOVA methods with SPSS 11.5 software (SPSS Inc., Chicago, IL, USA). $\mathrm{P}<0.05$ was considered to indicate a statistically significant difference.

\section{Results}

Maggot extract promoted wound healing. All together, 72 pieces of tracing message were collected from 8 open skin wounds at 9 time points in the control and 58 pieces from the experimental group respectively, which were totally documented in one transparency model sheet (Fig. 1A). The repairing rates of the skin wounds without and with maggot extract treatments were sequentially evaluated by transparency tracing-digital calculation method (12). As shown in Fig. 1B, the average unrepaired areas of group 1 dressed only with vaseline were larger than that of group 2 treated by maggot extract at any observation time points $(\mathrm{P}<0.05$; t-test). Two of eight wounds in group 2 completely closed at day 12 , four at day 14 and the last two at day 16; in contrast, all eight wounds in group 1 remained open at day 16 (Fig. 1A).

No effect of maggot extract on TUNEL-negative cell death Distinct cell death was observed in the wound tissues biopsied at the next two days (day 1 and day 2), which became attenuate at day 3 after the trauma irrespective to maggot extract treatment (Fig. 2A). To scrutinize the feature of the cell death, TUNEL assay was performed on those tissues, which revealed the rarity of TUNEL-positive cells in the regions with extensive cell death at the early wound stage (Fig. 2A).

Maggot extract reduced apoptosis in wound tissues. As shown in Fig. 2B, the dead cells in small sizes were common in the wound beds. TUNEL-positive cells (insets in Fig. 2B) were more frequently observed in the control group from day 3 in the average apoptosis rate of 47/vision field (X 40), became most remarkable at day 6 (56/vision field) and subsided thereafter in the time related fashion (Fig. 2C). The incidence of apoptotic cells were lesser common in the wound tissues treated by maggot extract at the corresponding time points $(\mathrm{P}<0.05)$ and became uncommon after day 9 of the trauma.

Maggot extract-enhanced BAG3 and Bcl-2 expression. The results of immunohistochemical staining (Fig. 3A)
A

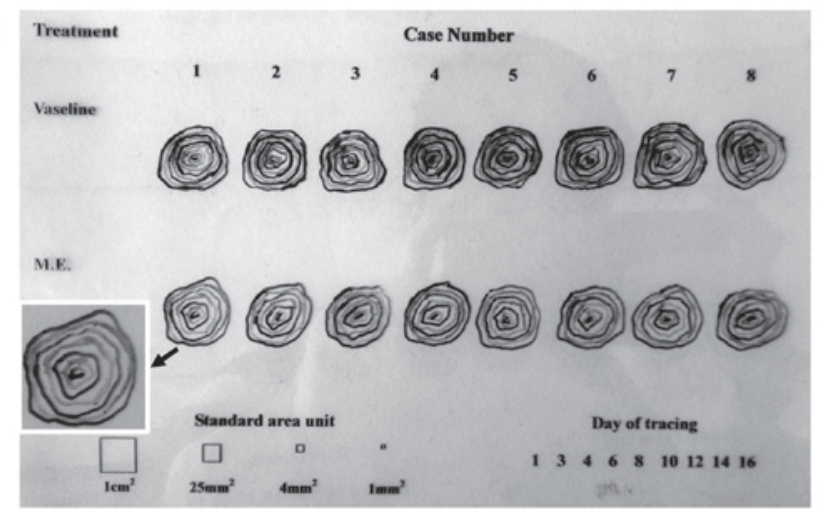

B

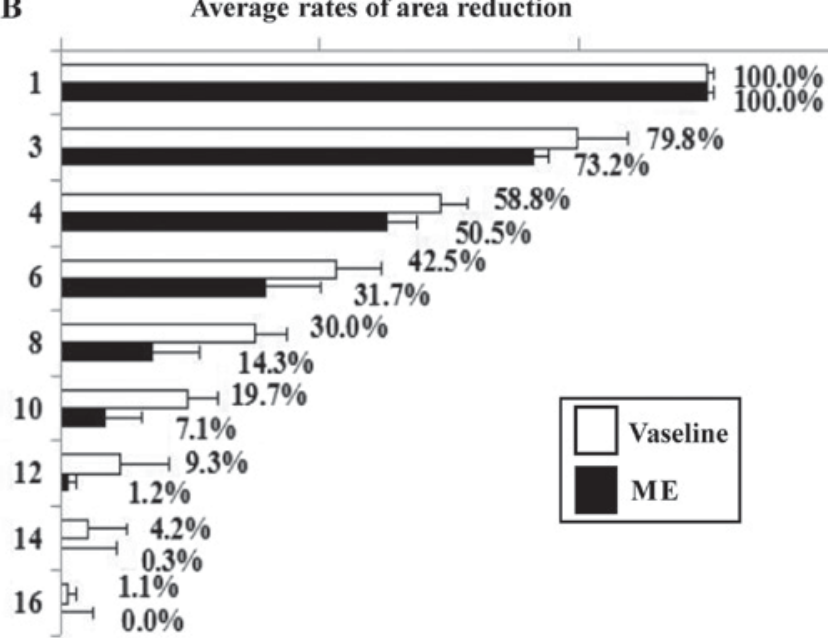

Figure 1. Evaluation of wound healing-promoting efficacy of maggot extract $(150 \mu \mathrm{g} / \mathrm{ml})$ (A). Representative transparency model sheet with complete traced massage of individual wound margins at nine observation time points (days 1, 3, 4, 6, 7, 10, 12, 14 and 16) after one of the three round experiments. Group 1: The wounds addressed with Vaseline only; group 2: The wounds treated by the extracts from the maggots without excretion/secretion. Arrow indicates the tracing message in higher magnification (B). Average repairing rates according to area calculation performed on the transparency tracings marked out from the wounds of the two experimental groups $(n=8$ wounds/group X 3) at nine observation times.

demonstrated that the level of BAG3 was expressed in low levels in the wound tissues of the control group, which was increased in maggot extract treated tissues. The immunohistochemical staining pattern of Bcl-2 was similar with that of BAG3 in terms of its elevated level after maggot extract treatment, especially at day 5. The results of western blotting for BAG3 and Bcl-2 were in accordance with that of immunohistochemical staining, showing increased production of BAG3 and Bcl-2 proteins in maggot extract treated tissues (Fig. 3B).

Beclin 1 and LC3 upregulation in extract-treated wound tissues. As shown in Fig. 3A and B, Beclin 1 was weakly expressed in the wound tissues and was upregulated following maggot extract treatment especially in the first week after the trauma. LC3 as another autophagy-associated factor was upregulated as well in the extract-treated wound tissues in the pattern as similar as Beclin 1. Western blotting demonstrated two LC3 bands in the molecular weights about $18 \mathrm{kDa}$ and $16 \mathrm{kDa}$, indicating the presence of the original (type-I) and enzymaticaly cleaved active form (type-II) of LC3 protein. The 
A

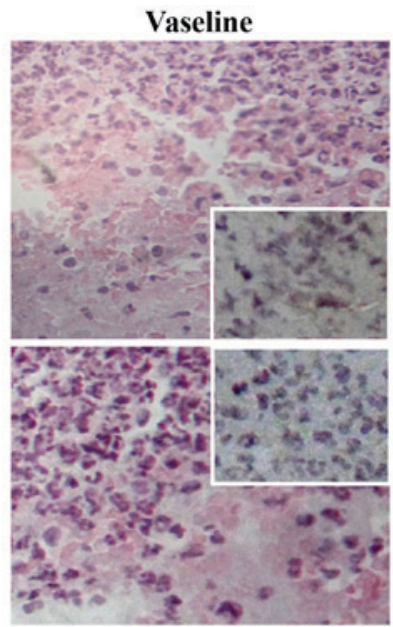

B
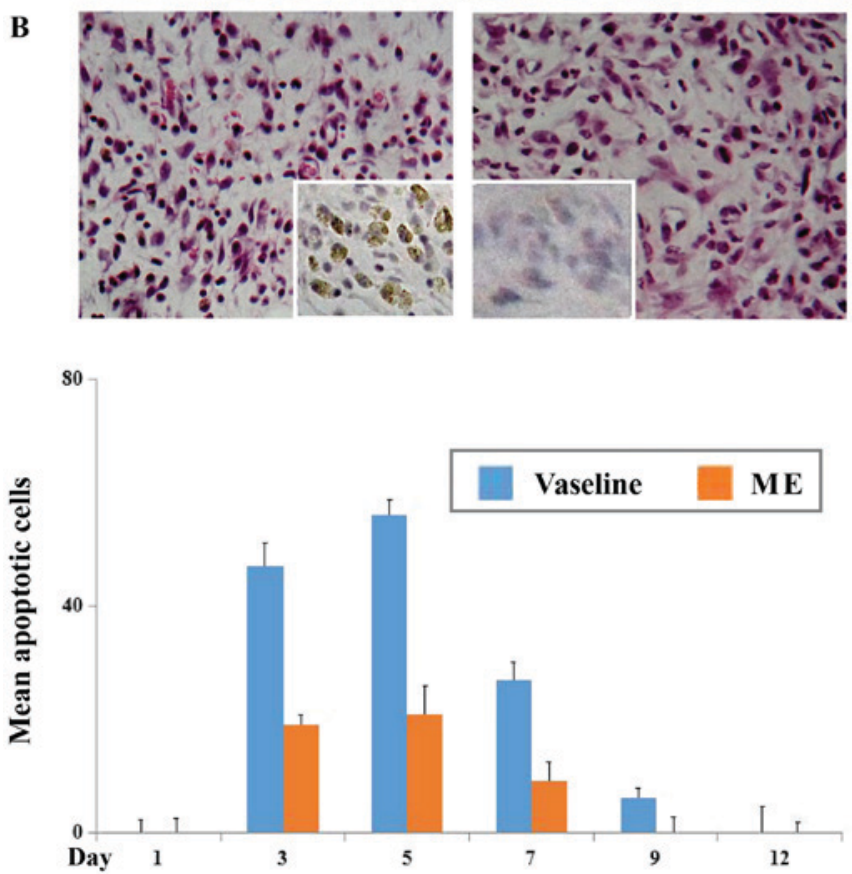

Figure 2. Effects of maggot extract on the cell death in the wound regions (A) No effect of maggot extract on TUNEL-negative cell death starting at day 1 and last to day 3 in the wound regions. The insets show that TUNEL-positive cells are rare in the wound tissues treated with vaseline and maggot extract (M.E.) (B). Comparison of the detection frequencies of TUNEL-positive cells in the wound tissues without (Vaseline) and with maggot extract treatment (M.E.) at day 5 after the trauma. Distinct difference of apoptosis indexes between the wound tissues of the two experimental groups. *, with statistical significance $(\mathrm{P}<0.05)$.

fraction of LC3 II was increased in the maggot extract-treated samples, especially those collected at day 3 , day 5 and day 8 time points (Fig. 3B).

\section{Discussion}

Acute and chronic skin wounds are the common injuries in clinics and the aim of their treatments is to reduce patients suffering through promoting the reconstruction of damaged tissues (29). Wound healing is stepwise processes with closely orchestrated biochemical events/cascades (30) in which the damaged or unhealthy cells are removed in the forms of necrosis and/or apoptosis, accompanied with the repair of lost tissues via active proliferation of the intact cell components.
A
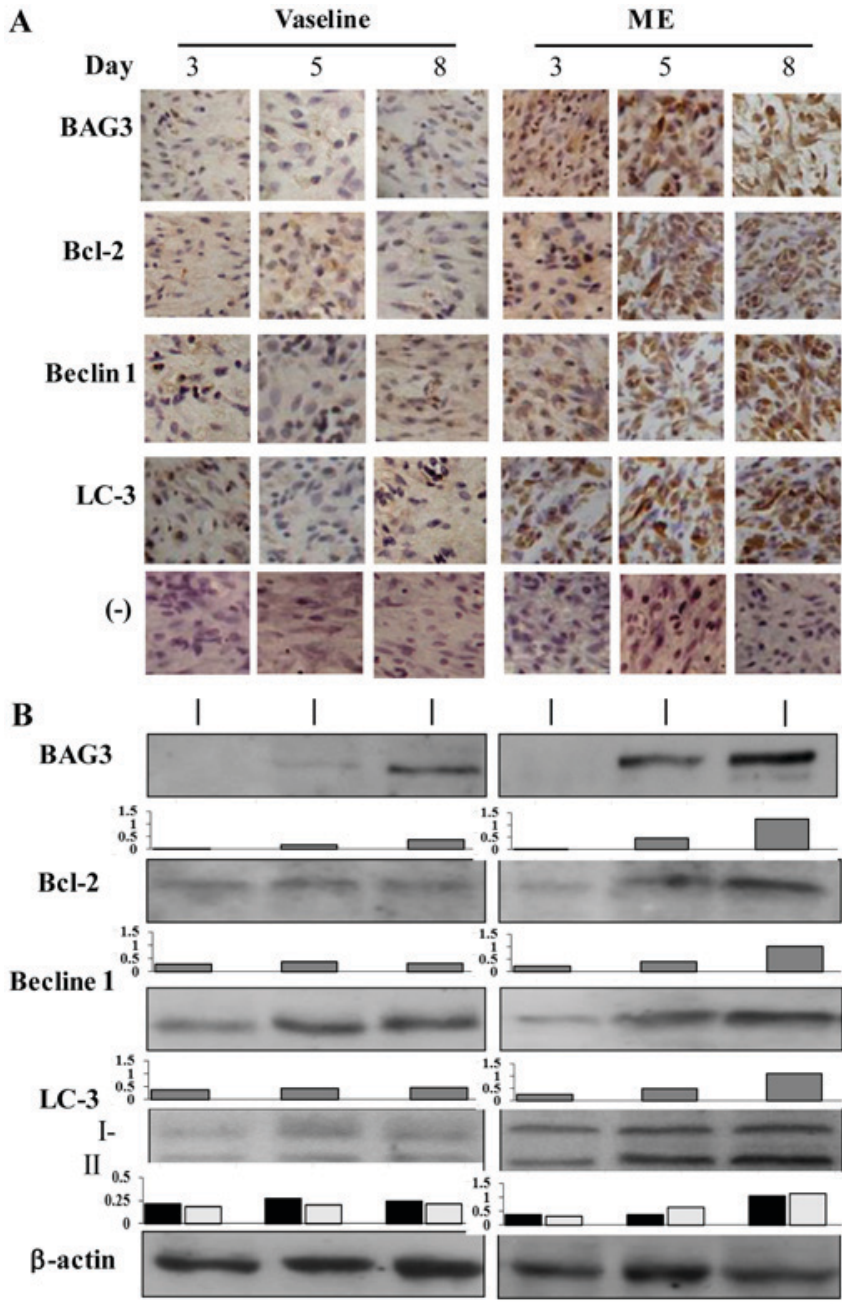

Figure 3. BAG3, Bcl-2, Beclin 1 and LC 3 expression in the wound tissues treated by vaseline only and maggot extract at the time points of day 3 , day 5 and day 8 ( $\mathrm{n}=8$ wounds/group X 3) (A). Immunohistochemical demonstration of BAG3, Bcl-2, Beclin 1 and LC 3 expression in the wound tissues treated by vaseline only (Vaseline) and maggot extract (M.E.). Enhanced expression of those genes is found in the maggot extract treated tissues. The corresponding tissue sections without 1st antibody incubation were used as negative controls (-) (B). Western blotting combined with grayscale analyses for BAG3, Bcl-2, Beclin 1 and LC3 expression and fractionation of LC3-I (18 kDa) and LC3-II $(16 \mathrm{kDa})$ in the wound tissues without (Vaseline) and with maggot extract treatment (M.E.).

Apparently, a well balanced cell loss and gain is essential for normal wound healing. Because the full maggot extract efficiently shortens the tissue closure time of rat skin wounds, we speculate that this natural bioactive mixture may also exert certain impact(s) on the cell death and maintenance in the wound tissues, although the effective components in the extract remain to be identified. Addressing these issues would provide further evidence for the practical use of maggot extract in the skin wound managements.

In the acute wound tissues, two types of cell death can be observed, they are, as demonstrated in the present study, necrosis due to cell damage and apoptosis induced by death signal-activated intrinsic suicide program (31). Although maggot extract shortens the wound closing time by promoting cell growth (12), it fails to rescue the cells from necrosis at the early days of skin damage, suggesting that this type of cell death is caused by direct physical damage or severe ischemia 
status (32) and is therefore unavoidable and irreversible. On the other hand, maggot extract effectively decrease the incidences of TUNEL-positive cells especially at the proliferation phase, indicating the reduced apoptotic pressure and enhanced anti-apoptosis activities in its treated wound tissues. Alternatively, the expression of anti-apoptosis factors may be upregulated by maggot extract, which prevent the cells from apoptosis through improving cell maintenance environment in the wound tissues and/or blocking the intracellular cascade of death signal transduction.

Many factors in the wound tissues can trigger the programmed cell death, of which cytochrome $\mathrm{C}$ and ROS released from mitochondrium are important death signals (33). Bax and Bak are pro-apoptosis proteins, which cause the increased permeability of mitochondrial membrane (34). Therefore, an inhibitor that suppresses Bax and Bak actions can stabilize the permeability of mitochondrial membrane and prevent the initiation of suicide program in the stressed cells. Bcl-2 is such inhibitor, because it is localized to the outer membrane of mitochondria, where it inhibits the apoptosis-promoting effects of Bak and Bax and sequesters the procaspases 8 and $9(35,36)$. BAG3 is another apoptosis-preventive protein and its knockdown leads to apoptosis-associated lower leg venous ulcers (25). Interestingly, BAG3 synergizes the anti-apoptotic effect of Bcl-2 (37). However, the statuses of Bcl-2 and BAG3, their impacts on apoptotic activity in wound tissues and their relevance with maggot extract promoted wound healing remain largely unknown. Our results demonstrate the upregulated BAG3 and Bcl-2 expression accompanied with reduction of apoptotic incidence in maggot extract treated tissues, suggesting the involvement of BAG3 and Bcl-2 in maggot extract inhibited apoptosis. It would also be possible that, in additional to improving cell proliferation environment, maggot extract inhibits apoptosis during wound healing through upregulating BAG3 and Bcl 2 expression. These findings further prove the efficacy of maggot extract in the treatment of skin wounds from the aspect of apoptosis regulation.

Autophagy is an adaptive response to environmental stresses including nutrient starvation due to tissue destruction or blood shortage $(38,39)$. Autophagic activity is closely associated with the levels of Beclin 1 expression and LC3 II generated by enzymatic cleavage (40). Increasing evidence reveals that BAG3 promotes autophagy via association with Beclin $1(24,41)$. However, no report has been so far available concerning the status of autophagy in acute skin wounds and its relevance to maggot extract promoted wound healing. We find that Beclin 1 and LC3 II production is increased in maggot extract treated tissues. Importantly, the cells with enhanced Beclin 1 and LC3 co-labeling are abundant in the tissues with reduced apoptotic incidence and BAG3 upregulation. These phenomena provide a cue to elucidate the potential cell protective effects of autophagy in the wound tissues to overcome nutrient shortage before the nutritional system has not been re-established in the regenerating tissues. The reduction of Beclin 1 and LC3 expression in the later stage of wound healing would support this notion. In this context, maggot extract regulated autophagic activity in the wound healing processes may be an attempt to prevent the intact cells from apoptosis rather than an additional cause of death.
Taken together, the results of the present study show remarkable apoptosis and low autophagy activity in the early stage of acute skin wound healing. Maggot extract, though failing to inhibit necrosis, efficiently facilitates wound closure and reduces the extent of apoptosis presumably through upregulating Bacl-2 expression. The elevated Beclin 1 expression and LC3 II fraction in maggot extract treated wound tissues indicate the enhanced autophagic activity. BAG3 is up-regulated by maggot extract, which may exert anti-apoptotic and autophagic effects in the wound tissues by interaction with Bcl-2 and Beclin 1, respectively. The above findings thus provide further cellular and molecular evidence for the effectiveness of maggot extract in local care of skin wounds. The above conclusion would be further strengthened by an experiment in which larvae are applied to the animal wound healing model mimicking a clinical treatment, followed by assessment of the parameters checked in the present study.

\section{Acknowledgements}

The present study was supported by the grants from National Natural Science Foundation of China (No. 81272786) and the special research fund for outstanding scholar of Dalian Medical University to Dr. Jia Liu. We thank the staffs in the Animal Center of Dalian Medical University for their humane care of the rats used this study during and after the experiments.

\section{References}

1. Eming SA, Martin P and Tomic-Canic M: Wound repair and regeneration: Mechanisms, signaling, and translation. Sci Transl Med 26: 265sr6, 2014.

2. Leavitt T, Hu MS, Marshall CD, Barnes LA, Lorenz HP and Longaker MT: Scarless wound healing: Finding the right cells and signals. Cell Tissue Res 365: 483-493, 2016.

3. Pereira RF and Bártolo PJ: Traditional therapies for skin wound healing. Adv Wound Care (New Rochelle) 5: 208-229, 2016.

4. Gottrup F, Jørgensen B and Karlsmark T: News in wound healing and management. Curr Opin Support Palliat Care 3: 300-304, 2009.

5. Reinke JM and Sorg H: Wound repair and regeneration. Eur Surg Res 49: 35-43, 2012 .

6. Johnson A and DiPietro LA: Apoptosis and angiogenesis: An evolving mechanism for fibrosis. FASEB J 27: 3893-3901, 2013.

7. Landén NX, Li D and Ståhle M: Transition from inflammation to proliferation: A critical step during wound healing. Cell Mol Life Sci 73: 3861-3885, 2016.

8. Ali N, Hosseini M, Vainio S, Taïeb A, Cario-André $M$ and Rezvani HR: Skin equivalents: Skin from reconstructions as models to study skin development and diseases. Br J Dermatol 173: 391-403, 2015.

9. Cappuzzello C, Doni A, Dander E, Pasqualini F, Nebuloni M, Bottazzi B, Mantovani A, Biondi A, Garlanda C and D'Amico G: Mesenchymal stromal cell-derived PTX3 promotes wound healing via fibrin remodeling. J Invest Dermatol 136: 293-300, 2016.

10. Walraven M, Beelen RH and Ulrich MM: Transforming growth factor- $\beta$ (TGF- $\beta$ ) signaling in healthy human fetal skin: A descriptive study. J Dermatol Sci 78: 117-124, 2015.

11. Honma M, Minami-Hori $M$, Takahashi $\mathrm{H}$ and Iizuka $\mathrm{H}$ : Podoplanin expression in wound and hyperproliferative psoriatic epidermis: Regulation by TGF- $\beta$ and STAT- 3 activating cytokines, IFN- $\gamma$, IL-6, and IL-22. J Dermatol Sci 65: 134-140, 2012.

12. Li PN, Li H, Wu ML, Wang SY, Kong QY, Zhang Z, Sun Y, Liu J and Lv DC: A cost-effective transparency-based digital imaging for efficient and accurate wound area measurement. PLoS One 7: e38069, 2012

13. Sano S, Chan KS and DiGiovanni J: Impact of Stat3 activation upon skin biology: A dichotomy of its role between homeostasis and diseases. J Dermatol Sci 50: 1-14, 2008. 
14. Jacobs AT and Marnett LJ: HSF1-mediated BAG3 expression attenuates apoptosis in 4-hydroxynonenal-treated colon cancer cells via stabilization of anti-apoptotic Bcl-2 proteins. J Biol Chem 284: 9176-9183, 2009.

15. Karpel-Massler G, Shu C, Chau L, Banu M, Halatsch ME, Westhoff MA, Ramirez Y, Ross AH, Bruce JN, Canoll P and Siegelin MD: Combined inhibition of Bcl-2/Bcl-xL and Usp9X/Bag3 overcomes apoptotic resistance in glioblastoma in vitro and in vivo. Oncotarget 6: 14507-14521, 2015.

16. Behl C: BAG3 and friends: Co-chaperones in selective autophagy during aging and disease. Autophagy 7: 795-798, 2011.

17. Arimura T, Ishikawa T, Nunoda S, Kawai S and Kimura A: Dilated cardiomyopathy-associated BAG3 mutations impair Z-disc assembly and enhance sensitivity to apoptosis in cardiomyocytes. Hum Mutat 32: 1481-1491, 2011.

18. Behl C: Breaking BAG: The Co-chaperone BAG3 in health and disease. Trends Pharmacol Sci 37: 672-688, 2016.

19. Merabova N, Sariyer IK, Saribas AS, Knezevic T, Gordon J, Turco MC, Rosati A, Weaver M, Landry J and Khalili K: WW domain of BAG3 is required for the induction of autophagy in glioma cells. J Cell Physiol 230: 831-841, 2015.

20. Menon J: Maggot therapy: A literature review of methods and patient experience. Br J Nurs 21: S38-S42, 2012.

21. Sherman RA: Mechanisms of maggot-induced wound healing: What do we know, and where do we go from here? Evid Based Complement Alternat Med 2014: 592419, 2014.

22. Li PN, Li H, Zhong LX, Sun Y, Yu LJ, Wu ML, Zhang LL, Kong QY, Wang SY and Lv DC: Molecular events underlying maggot extract promoted rat in vivo and human in vitro skin wound healing. Wound Repair Regen 23: 65-73, 2015.

23. Dixon BJ, Chen D, Zhang Y, Flores J, Malaguit J, Nowrangi D, Zhang $\mathrm{JH}$ and Tang J: Intranasal administration of interferon beta attenuates neuronal apoptosis via the JAK1/STAT3/BCL-2 pathway in a rat model of neonatal hypoxic-ischemic encephalopathy. ASN neuro 8: pii: 1759091416670492, 2016.

24. Rosati A, Graziano V, De Laurenzi V, Pascale M and Turco MC: BAG3: A multifaceted protien that regulates major cell pathways. Cell Death Dis 2: e141, 2011

25. Campitiello N, Faenza M, Pagliara D, Baldi C, Zeppa P, Rosati A and Rubino C: Expression of the anti-apoptotic BAG3 protein in leg venous ulcerative tissues. Cell Death Discov 2: 15068, 2016.

26. Lundberg C and Gerdin B: The role of histamine and serotonin in the inflammatory reaction in an experimental model of open wounds in the rat. Scand J Plast Reconstr Surg 18: 175-180, 1984

27. Li H, Chen XY, Kong QY and Liu J: Cytopathological evaluations combined RNA and protein analyses on defined cell regions using single frozen tissue block. Cell Res 12: 117-121, 2002.
28. Zhong LX, Zhang Y, Wu ML, Liu YN, Zhang P, Chen XY, Kong QY, Liu J and Li H: Resveratrol and STAT inhibitor enhance autophagy in ovarian cancer cells. Cell Death Discov 2: 15071, 2016.

29. Greaves NS, Iqbal SA, Hodgkinson T, Morris J, Benatar B, Alonso-Rasgado T, Baguneid M and Bayat A: Skin substitute-assisted repair shows reduced dermal fibrosis in acute human wounds validated simultaneously by histology and optical coherence tomography. Wound Repair Regen 23: 483-494, 2015.

30. Gurtner GC and Chapman MA: Regenerative medicine: Charting a new course in wound healing. Adv Wound Care (New Rochelle) 5: 314-328, 2016.

31. Rosińczuk J,TaradajJ,DymarekR and Sopel M: Mechanoregulation of wound healing and skin homeostasis. Biomed Res Int 2016: $3943481,2016$.

32. Johnson A and DiPietro LA: Apoptosis and angiogenesis: An evolving mechanism for fibrosis. FASEB J 27: 3893-3901, 2013.

33. Moseley R, Hilton JR, Waddington RJ, Harding KG, Stephens P and Thomas DW: Comparison of oxidative stress biomarker profiles between acute and chronic wound environments. Wound Repair Regen 12: 419-429, 2004.

34. Luna-Vargas MP and Chipuk JE: Physiological and pharmacological control of BAK, BAX, and beyond. Trends Cell Biol 26: 906-917, 2016.

35. O'Neill KL, Huang K, Zhang J, Chen Y and Luo X: Inactivation of prosurvival $\mathrm{Bcl}-2$ proteins activates $\mathrm{Bax} / \mathrm{Bak}$ through the outer mitochondrial membrane. Genes Dev 30: 973-988, 2016.

36. Luna-Vargas MP and Chipuk JE: The deadly landscape of pro-apoptotic BCL-2 proteins in the outer mitochondrial membrane. FEBS J 283: 2676-2689, 2016.

37. Tahrir FG, Knezevic T, Gupta MK, Gordon J, Cheung JY, Feldman AM and Khalili K: Evidence for the role of BAG3 in mitochondrial quality control in cardiomyocytes. J Cell Physiol 232: 797-805, 2017.

38. Takagi A, Kume S, Maegawa $\mathrm{H}$ and Uzu T: Emerging role of mammalian autophagy in ketogenesis to overcome starvation. Autophagy 12: 709-710, 2016.

39. Kaushal GP and Shah SV: Autophagy in acute kidney injury. Kidney Int 89: 779-791, 2016.

40. Tanida I: Autophagosome formation and molecular mechanism of autophagy. Antioxid Redox Signal 14: 2201-2214, 2011.

41. Gamerdinger M, Carra S and Behl C: Emerging roles of molecular chaperones and co-chaperones in selective autophagy: Focus on BAG proteins. J Mol Med (Berl) 89: 1175-1182, 2011. 